\title{
La risa contagiosa como vocalización provocadora de expresiones faciales y electromiográficas relacionadas con emociones positivas en los oyentes
}

\author{
Guillermo Arévalo-Pachón; Julio Eduardo Cruz
}

Cómo citar este artículo:

Arévalo-Pachón, G., \& Cruz, J. E. (2021). La risa contagiosa como vocalización provocadora de expresiones faciales y electromiográficas relacionadas con emociones positivas en los oyentes. Acta Colombiana de Psicología, 24(2), 45-58. https:// www.doi.org/10.14718/ACP.2021.24.2.5

Recibido, diciembre 01/2020; Concepto de evaluación, abril 07/2021; Aceptado, abril 29/2021

\author{
Guillermo Arévalo-Pachón ${ }^{1}$ \\ ORCID: https://orcid.org/0000-0002-3753-9144 \\ Universidad de los Andes, Bogotá, Colombia. \\ Julio Eduardo Cruz \\ ORCID: https://orcid.org/0000-0001-9431-8762 \\ Universidad de los Andes, Bogotá, Colombia.
}

\begin{abstract}
Resumen
A pesar de su relevancia para la comprensión de la expresión emocional vocal, el estudio de la risa contagiosa se encuentra en sus primeras etapas de investigación y aún no se ha establecido su naturaleza ni la de las respuestas que esta provoca. Teniendo esto en cuenta, el propósito de este estudio fue determinar si los estímulos acústicos de risa contagiosa, además de generar conductas de risa o sonrisa, provocan en los oyentes las expresiones faciales, electromiográficas y cardíacas de una emoción positiva. Para esto, se contó con la participación de 60 universitarios de ambos sexos con edades entre los 18 y los 30 años en un diseño experimental intrasujeto con mediciones en la condición de línea de base y en exposiciones a diferentes estímulos de risa contagiosa, donde se verificaron tres hipótesis en las que se comparó expresiones faciales de alegría (medidas con el software FaceReader), amplitud electromiográfica (EMG) del músculo cigomático mayor (medida con el módulo EMG-100 del Biopac) e intervalos R-R como indicadores de frecuencia cardíaca (medidos con el módulo ECG-100 del Biopac) entre las diferentes condiciones. Como resultado, se encontraron diferencias significativas en los porcentajes de las expresiones faciales de alegría y amplitud EMG del cigomático al comparar las condiciones de línea de base y estímulos de risa más contagiosa, y de risas más y menos contagiosas; no obstante, no se encontraron diferencias significativas en los intervalos R-R en ninguna de las condiciones comparadas. Como conclusión, se comprobó la naturaleza emocional positiva de la risa/sonrisa provocada por estímulos de risa contagiosa y la proporcionalidad entre la intensidad de las expresiones faciales y las respuestas EMG elicitadas por esta risa y el grado de contagio percibido de la misma.

Palabras clave: risa, contagio emocional, alegría, expresión facial, electromiografía facial.
\end{abstract}

\footnotetext{
1 Cr. 1a\#18a-12, Universidad de los Andes, Bogotá, Colombia. Tel.: (571)3394949, ext. 2578. C. P.: 111711.g.arevalo10@uniandes.edu.co En este trabajo se reportan los resultados del segundo estudio de disertación doctoral (no publicado) de Guillermo Arévalo Pachón. Universidad de los Andes.
} 


\title{
Contagious laughter as a provocative vocalization of facial and electromyographic expressions related to positive emotions in listeners
}

\begin{abstract}
Despite its relevance for the understanding of vocal emotional expression, the study of contagious laughter is in its early stages of research and neither its nature nor the nature of the responses it provokes have not yet been established. The purpose of this study was to determine whether the acoustic stimuli of contagious laughter, in addition to generating laughter or smiling behaviors, elicit in listeners the facial, electromyographic and heart expressions of a positive emotion. To this end, 60 university students of both sexes, aged between 18 and 30 years participated in an intrasubject experimental design with measurements in the baseline condition and in exposures to different contagious laughter stimuli, where three hypotheses were tested comparing facial expressions of joy (measured with the FaceReader software), electromyographic amplitude (EMG) of the zygomaticus major muscle (measured with the Biopac EMG-100 module) and RR intervals as indicators of heart rate (measured with the Biopac ECG-100 module) between the different conditions. As a result, significant differences were found in the percentages of facial expressions of joy and zygomatic EMG amplitude when comparing the baseline and stimulus conditions of more contagious laughter, and more and less contagious laughter. However, no significant differences were found in the R-R intervals in any of the compared conditions. In conclusion, the positive emotional nature of laughter / smile caused by contagious laughter stimuli and the proportionality between the intensity of the facial expressions and EMG responses elicited by this laughter and the perceived degree of contagiousness of laughter were verified. Keywords: laughter, emotional contagiousness, joy, facial expression, facial electromyographynt.
\end{abstract}

\section{Introducción}

La risa es una señal comunicativa no verbal y multidimensional que influye de manera importante en las interacciones sociales (Consentino et al., 2016). Por varias décadas, la investigación sobre risa se centró especialmente es su expresion facial, para diferenciar las risas espontáneas de las voluntarias (Gervais \& Wilson, 2005); sin embargo, en los últimos años se han realizado investigaciones sobre los aspectos acústicos, perceptuales, conductuales y cerebrales de esta vocalización y de las diferentes modalidades de risa (p. ej., Szameitat et al., 2009a; Szameitat et al., 2009b, Szameitat et al., 2010; Wood et al., 2017).

Una de las múltiples modalidades que puede presentar esta vocalización es la risa contagiosa, y fue Provine (1992) quien realizó la primera investigación empírica sobre esta risa, en la cual se comprobó que la exposición a audios de esta vocalización elicita de forma automática conductas de risa o sonrisa en la mayoría de los oyentes, y que estas tienen características de respuestas estereotipadas típicas de la especie. Sin embargo, la investigación sobre risa contagiosa no ha tenido el desarrollo esperado (Provine, 2015), y por ello no se ha establecido la naturaleza de la misma ni la de las risas o sonrisas provocadas por ella.

En nuestro medio encontramos un antecedente empírico sobre este tipo de risa, pues el primer estudio de la investigación de Arévalo (2020) sobre risa contagiosa $(N=132)$ determinó la percepción de contagio de 66 risas y la relación con sus parámetros acústicos, y en ese trabajo se estableció que: (a) algunos parámetros acústicos permiten predecir la calificación de contagio de esta risa; (b) la duración de los estímulos de risa influyen en su percepción de contagio; (c) no hay diferencias de sexo en la apreciación de contagio de diversos estímulos femeninos o masculinos de risa contagiosa; y (d) hay diferencias significativas en la apreciación de contagio entre las risas calificadas como más y menos contagiosas. En dicha investigación se definió la risa más contagiosa como aquella vocalización femenina o masculina que recibe las calificaciones de contagio más altas, y la menos contagiosa como aquella risa de cualquier sexo que recibe las calificaciones de contagio más bajas. Dado que en el presente estudio se utilizan algunos de los estímulos evaluados en dicha investigación, se optó por adoptar estas definiciones sobre las diferentes risas.

Ahora bien, sabemos que la risa humana puede desempeñar una o dos de las siguientes funciones: ser una señal no verbal de interacción social, o ser una forma de expresión emocional (Consentino et al., 2016). Por este motivo, es posible que algunas risas no posean naturaleza emocional, como aquellas que se emiten en situaciones sociales para indicar acuerdo o cortesía (Neves et al., 2018). Asimismo, es importante mencionar que las risas que se acompañan de emociones positivas se originan en regiones cerebrales específicas —amígdala, áreas talámicas e hipotalámicas y el área dorsal-tegmental del tallo cerebral—, que tienen características acústicas y faciales distintivas, y que se perciben como espontáneas (Gervais \& Wilson, 2005). Así, para conocer la naturaleza de una determinada risa, resulta relevante establecer si esta se puede considerar o no como una auténtica expresión emocional. 
Ante la posibilidad de ampliar el conocimiento sobre la naturaleza de las respuestas provocadas por la risa contagiosa, su relación con los estímulos elicitadores, los alcances del contagio y sus posibles relaciones con otros fenómenos psicológicos - p. ej., influencia y cohesión social, empatía, expresión emocional innata, etc.- - para la presente investigación se tuvo el objetivo de determinar si los estímulos acústicos de risa contagiosa, además de generar conductas de risa o sonrisa (Provine, 1992), provocan en los oyentes las expresiones faciales, electromiográficas y cardíacas de una emoción positiva.

Inicialmente, es importante saber que la presunta naturaleza emocional de la risa o sonrisa provocadas por la risa contagiosa se puede enmarcar en dos aproximaciones teóricas: la primera se refiere a la capacidad de la risa con determinados atributos acústicos para provocar respuestas emocionales, afectivas y conductuales en los oyentes (p. ej., Owren et al., 2013); y la segunda es la teoría del contagio emocional, que considera que el estado emocional de una persona puede influir en las conductas y emociones de otra u otras personas (Ruef-López et al., 2015), y que el proceso de contagio emocional implica las etapas de imitación, retroalimentación y contagio que pueden producir convergencia conductual, vocal y emocional entre los participantes de la interacción (Hatfield et al., 2014). No obstante, el contagio a través de vocalizaciones no ha sido suficientemente investigado (Ruef-López et al., 2015), por lo cual el presente estudio podría aportar evidencia indirecta sobre este tema.

Ahora bien, dado que la risa/sonrisa provocada por la risa contagiosa es generada por un estímulo antecedente espontáneo de valencia positiva, una de las condiciones para generar risa emocional - o risa Duchenne-(Gervais \& Wilson, 2005; Whalen, 2010) es que esta sea una risa emocional de naturaleza positiva. Teniendo esto en cuenta, entre todas las posibles emociones que pueden acompañar la risa/sonrisa provocada, en la presente investigación se seleccionó la alegría, pues esta emoción tiene algunas características compatibles con las reacciones y funciones que se le atribuyen a la risa contagiosa: (a) ambas son evaluadas positivamente tanto por el emisor como por el receptor de la misma, (b) ambas facilitan el acoplamiento emocional y conductual en los grupos, y (c) las dos fomentan la creación de vínculos sociales (Szameitat et al., 2009b). De este modo, se asume que las respuestas provocadas por risa contagiosa podrían estar asociadas con esta emoción positiva.

No obstante, con base en las teorías que consideran que cada emoción se expresa en diferentes dimensiones que no siempre correlacionan (p. ej., Mauss et al., 2005), se ve la necesidad de obtener información de diferentes formas de expresión emocional, para que, de manera convergente, se pueda establecer si la alegría acompaña o no a las risas/sonrisas provocadas por estímulos acústicos de risa contagiosa.

Por otra parte, es necesario tener en cuenta que la expresión facial se ha considerado como una dimensión importante para determinar la presencia e intensidad de la emoción que experimenta una persona (Consentino et al., 2016), pero la literatura señala otras razones que justifican la inclusión de esta dimensión en la presente investigación, como que: (a) la alegría como emoción básica tendría una expresión facial distintiva y universal (p. ej., Matsumoto et al., 2008); (b) se ha identificado que la expresión facial que caracteriza inequívocamente a la risa que acompaña a las emociones positivas - o risa Duchenne- (p. ej., Whalen, 2010); (c) se ha evidenciado alta correlación entre la expresión emocional de la alegría —o felicidad-y la identificación de esta emoción por parte de observadores (p. ej., Matsumoto, 1992); y (d) la expresión emocional de la alegría es susceptible de medición cuantitativa y confiable (Consentino et al., 2016). Y es de esto que surge la primera hipótesis del estudio (H1): la exposición de los participantes del estudio a estímulos acústicos de risa más contagiosa provoca mayores expresiones faciales de alegría en comparación con las observadas en línea de base y ante estímulos de risa menos contagiosa.

De otro lado, es sabido que la electromiografía (EMG) facial es una técnica altamente confiable para detectar la existencia, valencia e intensidad de una emoción en sujetos expuestos a determinados estímulos, pues esta cuantifica la actividad eléctrica de la contracción muscular de ciertos músculos de la cara (Hess, 2009). De hecho, la EMG facial se ha considerado un método muy sensible para detectar emociones, pues es capaz de registrar actividad eléctríca de elicitadores fuertes o débiles, además de que permite discriminar confiablemente la presencia de emociones primarias a pesar de las diferencias individuales relacionadas con la morfología del rostro (Boxtel, 2010). Incluso, se ha propuesto que las señales EMG pueden ayudar a determinar con mayor seguridad si la risa contagiosa se relaciona con la alegría, pues estas no solo detectan las expresiones emocionales observables, sino también las no observables (Cosentino et al., 2016). Así, se puede afirmar que esta técnica complementa y enriquece el reconocimiento de emociones que se realiza mediante el análisis de la expresión facial.

Adicional a esto, la literatura sobre el tema señala que el incremento de la actividad EMG superficial — de mayor amplitud - del músculo facial cigomático mayor se relaciona con emociones o estados de ánimo positivos (Boxtel, 2010); y, específicamente, se ha encontrado que 
los estímulos acústicos elicitadores de emociones positivas generan incremento en la actividad eléctrica de este músculo (Hietanen et al., 1998). Así, con base en estas evidencias, se formula la segunda hipótesis de trabajo (H2): la exposición de los participantes a estímulos de risa más contagiosa provoca mayor actividad EMG del músculo facial cigomático en comparación con la que se observa en la condición de línea de base y ante estímulos de risa menos contagiosa.

Finalmente, hay evidencia de que uno de los correlatos psicofisiologicos más importantes de la alegría son los cambios en la actividad cardiovascular - frecuencia cardíaca, presión sanguínea y volumen sanguíneo periférico- (Domínguez, 2013). Por ejemplo, la revisión de Kreibig (2010) sobre esta emoción señaló que la alegría se caracteriza por el incremento de la activación simpática adrenérgica, que se manifiesta por aumentos en la tasa cardíaca y ausencia de cambios en la conductancia de la piel; $y$, en general, la literatura señala que la alegría se acompaña de un leve aumento de la frecuencia cardíaca, que es más evidente cuando esta emoción se expresa con risa (p. ej., Domínguez, 2013). Incluso, hay razones que explican la elevación de la tasa cardíaca en episodios de risa, como que esta vocalización implica cambios en la frecuencia respiratoria, compresión de las vías respiratorias y gasto energético, todo lo cual altera la frecuencia cardíaca (Buchowski et al., 2007).

Teniendo esto último en cuenta, en este estudio se toman los intervalos R-R como indicador de frecuencia cardíaca - estos se refieren a la duración entre picos de ondas $\mathrm{R}$, que se relacionan con mayor actividad eléctrica en cada latido del corazón-: a mayor frecuencia cardíaca, menor duración de los mismos. Así, con base en estas consideraciones, surge la tercera hipótesis de esta investigación(H3): la exposición de los participantes a estímulos de risa más contagiosa provoca mayor frecuencia cardíaca - menor duración de los intervalos R-R- que la que se observa en la condición de línea de base y ante estímulos de risa menos contagiosa.

\section{Método}

\section{Tipo de estudio y diseño}

Se llevó a cabo un estudio de laboratorio con algunas características de los estudios experimentales, a excepción de la selección aleatoria de los participantes. Específicamente, para comprobar las hipótesis se utilizó un diseño intrasujeto (Shaughnessy et al., 2007) en el que cada participante fue evaluado en la condición preprueba y en las demás condiciones del factor intrasujeto - tipo de risa contagiosa: femenina y masculina más y menos contagiosa-, manteniendo control sobre las posibles variables de confusión - constancia en instrucciones, procedimiento, volumen de audios, condiciones ambientales y en la toma de mediciones y presentación aleatoria de estímulos, etc.- - Las variables dependientes fueron los porcentajes de expresión facial de alegría, la amplitud promedio de ondas EMG y la duración promedio de intervalos R-R.

\section{Estímulos}

Se tomaron cuatro estímulos acústicos de risa contagiosa con un mismo rango de duración — de 12 a 16 segundosdel primer estudio de la investigación de Arévalo (2020), calificados por 132 sujetos como risas femeninas y masculinas más y menos contagiosas. En dicha investigación se demostró empíricamente que las risas calificadas como más y menos contagiosas diferían significativamente en su apreciación de contagio $(t=11.88(5) ; p<.05)$, en la duración de las respuestas provocadas de risa o sonrisa (ANOVA de medidas repetidas $F(3,162)=25.77 ; p<.05$; $\mathrm{n}^{2}=0.32$ ), y en la frecuencia de provocación de respuestas de risas o sonrisas frente a la ausencia de respuestas (para risas femeninas: $\mathrm{McNemar}=24.14, p<.05$; $\mathrm{y}$ para risas masculinas: $\mathrm{McNemar}=8.04, p<.05$ ).

\section{Muestra}

El tamaño de la muestra se calculó con el software GPower, versión 3.1.9.2 (alfa: .05, tamaño de efecto: 0.33 y poder: .95) para una prueba de ANOVA de medidas repetidas con factores intra. Teniendo en cuenta lo encontrado allí, se tomó una muestra por conveniencia de 60 universitarios: 39 mujeres $(65 \%)$ y 21 hombres ( $35 \%$ ) de nivel de estudios de pregrado de la ciudad de Bogotá. Los criterios de inclusión de la muestra fueron que los participantes fueran estudiantes universitarios - de ambos sexos - con edades entre 18 y 30 años, y que reportaran buena salud, audición normal y visión normal o normal corregida.

\section{Instrumentos, equipos y softwares}

Para el desarrollo de este trabajo se hizo uso de: (a) el programa OpenSesame (versión 3.2.7 de 2018), que permitió diseñar y aplicar el experimento, asegurando alto grado de control y estandarización del procedimiento (Mathôt et al., 2012); (b) el Biopac Systems M150 (Inc. Santa Barbara, C. A.) - software AcqKnowledge y módulos EMG 100C y ECG 100-C-; (c) el Software Qualtrics (Qualtrics Provo, UT, versión 2018), una plataforma en línea que permite diseñar, distribuir y recolectar la información de encuestas; (d) el programa FaceReader (Noldus Information Technology), un software que analiza automáticamente 
las expresiones faciales de seis emociones básicas con alto grado de confiabilidad $-88 \%$ de confiabilidad en el reconocimiento general de emociones-, en el que, con base en los estudios que han utilizado este programa (p. ej., Maison \& Pawlowska, 2017), para el presente estudio se asumió la naturaleza continua de los datos que arroja el programa; (e) la plataforma de videos online Hippo Video (Lyceum Technologies, Pvt. Ltd.), que permitió crear videos de cada participante en cada condición con una resolución de 1080 pixeles; (f) una computadora personal HP DESKTOP-BETHT9R, con la cual se grabaron los videos de cada participante; y $(\mathrm{g})$ un computador portátil Hewlett Packard DESKTOP-4HRV3g6, mediante el cual se aplicó el experimento.

\section{Consideraciones éticas}

El análisis de las aspectos éticos del presente estudio concluyó que por el tipo de estímulos utilizados, la aplicación de medidas no invasivas y las condiciones de participación de manera informada, libre y en condiciones de respeto a la dignidad humana, la presente investigación representaba un riesgo mínimo para los participantes - un "riesgo que puede presentarse en estudios prospectivos que emplean el registro de datos a través de procedimientos comunes consistentes en: exámenes físicos o psicológicos de diagnóstico o tratamientos rutinarios, pruebas psicológicas a grupos o individuos en los que no se manipulará la conducta del sujeto”, según la Resolución 008430 de 1993 -. Adicionalmente, esta investigación recibió el aval del Comité de Ética de la universidad donde se realizó el estudio.

\section{Procedimiento}

Para el desarrollo del presente estudio se tuvieron en cuenta los siguientes pasos:

a. Diseño del experimento mediante el software OpenSesame, que implicó la estandarización de instrucciones, la presentación aleatoria de los estímulos de risa con una misma duración, y la toma de mediciones e intervalos entre ensayos dentro de un mismo rango de tiempo.

b. Implementación de estudio piloto, en el cual participaron seis universitarios diferentes a los que conformaron la muestra.

c. Invitación personal a los estudiantes universitarios para participar en la investigación, así como la obtención del consentimiento informado de aquellos que aceptaron voluntariamente ser parte del estudio. Tanto el consentimiento informado como la recolección de los datos demográficos de los participantes se obtuvieron mediante el software Qualtrics.

d. Aplicación del experimento diseñado mediante el software OpenSesame, así como la toma de datos de las variables dependientes seleccionadas. El experimento se llevó a cabo en un laboratorio de biorretroalimentación, con las condiciones de aislamiento y control de variables que requería el estudio ${ }^{1}$. Para registrar la actividad EMG y ECG se siguieron los protocolos del Manual del Biopac respecto a la ubicación de los electrodos y la toma de registros (Biopac Systems, Inc., 2016). Las expresiones faciales de los participantes fueron grabadas mediante el software Hippo Video mientras estos eran expuestos a las diferentes condiciones del estudio.

e. Recolección y procesamiento de datos. Cada uno de los videos de las diferentes condiciones a las que se expusieron los participantes se analizó mediante el software FaceReader. Con el software AcqKnowledge se recuperaron los datos recolectados mediante el Biopack y se hicieron las transformaciones recomendadas de los registros de amplitud EMG - expresados en voltios-y de los intervalos $\mathrm{R}-\mathrm{R}$ - expresados en fracciones de segundo-.

f. Análisis estadístico de los resultados. Teniendo en cuenta el diseño utilizado para comprobar cada una de las hipótesis, en los casos en que se compararon dos variables con distribución no normal se utilizó la prueba no paramétrica para muestras relacionadas de Wilcoxon, mientras que cuando se compararon las medias de las diferentes condiciones de risa femenina y masculina se utilizó un ANOVA de medidas repetidas. Dada la no normalidad de la mayoría de las variables utilizadas para establecer las diferencias de las expresiones emocionales según el sexo de los participantes, se utilizó la prueba de Wilcoxon.

g. Discusión de los resultados con base en las teorías y otros estudios empíricos sobre el tema.

\section{Resultados}

Para la comprobación de la primera hipótesis $(\mathrm{H} 1)$ de esta investigación —que afirmaba que la exposición de

\footnotetext{
1 Específicamente, con el equipo Biopack y los módulos EMG $100-\mathrm{C}$ y ECG 100-C se obtuvieron y analizaron las señales electromiográficas y electrocardiográficas superficiales, donde la configuración para el módulo EMG 100C fue: ganancia: 2000; LP: $500 \mathrm{~Hz}$; 100HzHp: Off; HP: $10 \mathrm{~Hz}$; y la configuración para el módulo ECG 100C fue: ganancia: 2000; Mode: NORM; 35HzLPN: On/35HzLPN; HP: $0.5 \mathrm{~Hz} / 1.0 \mathrm{~Hz}$.
} 
los participantes del estudio a estímulos acústicos de risa más contagiosa provoca mayores expresiones faciales de alegría en comparación con las observadas en línea de base y ante estímulos de risa menos contagiosa—, se comparó de forma independiente la condición de exposición a estímulos de risa más contagiosa vs. línea de base, seguida por la exposición a estímulos de risa menos contagiosa vs. línea de base. Los datos descriptivos relevantes para esta hipótesis se señalan en la Tabla 1.

Tabla 1.

Media, desviación típica e intervalos de confianza de los porcentajes de alegría en las diferentes condiciones experimentales

\begin{tabular}{cccc}
\hline Condición experimental & $M E$ & $D T$ & $I C$ \\
\hline Línea de base & 0.05 & 0.09 & $0.029-0.084$ \\
Risa femenina más contagiosa & 0.39 & 0.25 & $0.32-0.47$ \\
Risa masculina más contagiosa & 0.35 & 0.24 & $0.28-0.43$ \\
Risa femenina menos contagiosa & 0.19 & 0.25 & $0.12-0.27$ \\
Risa masculina menos contagiosa & 0.24 & 0.23 & $0.17-0.32$ \\
\hline
\end{tabular}

Nota.ME: media; $D T$ : desviación típica; $I C$ : intervalos de confianza.

Por un lado, para la comparación de las expresiones faciales de alegría en las condiciones de risa más contagiosa vs. línea de base se contó con un $N=53$. La prueba no paramétrica de rangos de Wilcoxon, usada debido a la ausencia de normalidad en la variable diferencia entre los porcentajes de las expresiones faciales de alegría en línea de base y ante audios de risa femenina más contagiosa (Shapiro-Wilks: $p<.05$ ) mostró que existen diferencias significativas entre ambas condiciones $(\mathrm{z}=-6.09, p<.05)$, siendo más altos los valores de la risa femenina más contagiosa. Además, se encontró que la diferencia de expresiones faciales de alegría entre los valores de la condición de línea de base y de risa masculina más contagiosa tenía distribución normal (Shapiro-Wilks: 0.95(49) $p>.05$ ). La prueba $t$ mostró diferencias significativas entre ambos porcentajes $(t(48)=-9.16, p<.05)$, siendo más altos los segundos que los primeros.
Por otro lado, para la comparación de las condiciones de exposición a risas más contagiosas vs. risas menos contagiosas se tuvo un $N=45$. Dada la robutez del ANOvA unifactorial de medidas repetidas ante la violación del supuesto de normalidad en algunas variables incluidas en el análisis (Blanca et al., 2017), la significación de esta hipótesis se estableció mediante esta prueba —el factor intrasujeto fue el de tipos de risa, y la variable de medida fue la de porcentajes de expresiones de alegría-. Los datos mostaron un valor de Mauchly significativo $(p>.05)$ y un valor de $F(3,389)=22.54, p<.05$, el cual señala la existencia de diferencias significativas en los porcentajes de expresiones de alegría ante audios de risa más y menos contagiosa. En la comparación por pares establecida mediante la prueba de Wilcoxon se encontraron diferencias significativas en las expresiones de alegría provocadas por las risas femeninas y masculinas más y menos contagiosas $(\mathrm{z}=-5.36(p<.05), \mathrm{y} \mathrm{z}=-3.59(p<.05)$, respectivamente $)$, y entre la risa femenina menos contagiosa y masculina más contagiosa $(\mathrm{z}=-4.67(p<0.05))$.

Así, con base en los datos señalados en los dos párrafos anteriores, se puede afirmar que la primera hipótesis $(\mathrm{H} 1)$ de este estudio recibió apoyo empírico, lo cual indica que hay incrementos significativos en la expresión facial de alegría en exposiciones a estímulos acústicos de risa más contagiosa, así como mayores niveles de esta emoción ante audios de risa más contagiosa que menos contagiosa.

Por otra parte, la comprobación de la segunda hipótesis (H2) de esta investigación — que refiere a que la exposición de los participantes a estímulos de risa más contagiosa provoca mayor actividad EMG del músculo facial cigomático en comparación con la que se observa en la condición de línea de base y ante estímulos de risa menos contagiosa—, se realizó analizando de forma independiente las dos comparaciones implicadas en la hipótesis. Para esto, debido a que ninguna de las variables analizadas en la comparación de los datos EMG entre línea de base y exposición a risas más contagiosas muestra una distribución normal (Kolmogorov-Smirnov: $p<.05)$, la significación estadística de esta hipótesis se

Tabla 2.

Prueba de rangos de Wilcoxon de promedio de amplitud EMG de los participantes en línea de base y frente a los estímulos de risa femenina y masculina más contagiosa

\begin{tabular}{|c|c|c|c|c|c|}
\hline Condición experimental & $\begin{array}{l}\text { Promedio de } \\
\text { amplitud EMG }\end{array}$ & $\begin{array}{c}\text { Intervalos de } \\
\text { confianza }\end{array}$ & $D T$ & Valor de Z & Significación \\
\hline Línea de base & 0.0068 & $0.0050-0.0085$ & 0.0068 & \multirow[b]{2}{*}{-6.62} & \multirow[b]{2}{*}{$p<0.05$} \\
\hline $\begin{array}{l}\text { Estímulo de risa femenina } \\
\text { más contagiosa }\end{array}$ & 0.0424 & $0.031-0.054$ & 0.0443 & & \\
\hline Línea de base & 0.0068 & $0.0050-0.0085$ & 0.0068 & \multirow[b]{2}{*}{-6.71} & \multirow[b]{2}{*}{$p<0.05$} \\
\hline $\begin{array}{l}\text { Estímulo de risa masculina } \\
\text { más contagiosa }\end{array}$ & 0.0316 & $0.024-0.040$ & 0.0307 & & \\
\hline
\end{tabular}


estableció mediante la prueba no paramétrica de rangos de Wilcoxon (véase Tabla 2).

Como se puede observar, la Tabla 2 señala la existencia de diferencias significativas en la amplitud EMG del músculo cigomático mayor provocada por los estímulos de risa femenina y masculina más contagiosas en comparación con los valores observados en línea de base, siendo más altos los primeros que los segundos. Estos resultados ofrecen apoyo a este componente de la segunda hipótesis (H2) de esta investigación.

De otro lado, la comparación de las respuestas EMG entre las diferentes condiciones de risa más y menos contagiosa se basó en los valores descriptivos de amplitud — expresados en voltios - de las risas más contagiosas señalados en la Tabla 2 y en los siguientes valores de las risas no contagiosas: (a) risa femenina menos contagiosa: $M E=0.0208$, $D T=0.0262, I C: 0.014-0.028 ; \mathrm{y}(\mathrm{b})$ risa masculina menos contagiosa: $M E=0.0212, D T=0.0236, I C: 0.015-0.027$.

Asimismo, la significación estadística de la diferencia de las medias entre las risas más y menos contagiosas se determinó mediante un ANOVA de medidas repetidas: el factor intrasujeto fue el tipo de risa, y la variable de medida fue la de promedios de amplitud EMG. Con esto, se encontró que el valor de Mauchly no fue significativo $\left(\chi^{2}=28.30\right.$, $p<.05$ ), por lo cual se tomó la corrección de GreenhouseGeisser, que mostró el siguiente valor de efectos intrasujetos: $F(2.33137 .76)=14.60, p<.05, \mathrm{n}^{2}=0.19$. Posteriormente, en la comparación por pares establecida mediante la prueba de Wilcoxon sí se encuentraron diferencias significativas en los promedios de amplitud ante las risas femeninas y masculinas más y menos contagiosas: $\mathrm{z}=-5.26(p<.05)$ $\mathrm{y} \mathrm{z}=-5.44(p<.05)$, respectivamente.

Además, en la comparación por pares se encontraron diferencias significativas entre la risa masculina más contagiosa y la femenina menos contagiosa $(\mathrm{z}=-4.66, p<.05)$, entre la masculina menos contagiosa y la femenina más contagiosa $(\mathrm{z}=-3.84, p<.05)$, y entre las masculinas más y menos contagiosas $(\mathrm{z}=-3.84, p<.05)$. Estos resultados señalan que existen diferencias significativas en las amplitudes EMG del músculo cigomático ante las diferentes risas a las que fueron expuestos los participantes.

En resumen, a nivel general los datos apoyan la segunda hipótesis (H2) formulada en la presente investigación, lo cual indica que la exposición a estímulos de risa más contagiosa elicita incrementos EMG en el músculo cigomático respecto a línea de base, y mayores valores EMG con respecto a los que provocan los audios de risa menos contagiosa.

Finalmente, la comprobación de la tercera hipótesis (H3) de este estudio — que refiere a que la exposición de los participantes a estímulos de risa más contagiosa provoca mayor frecuencia cardíaca (menor duración de los intervalos R-R) que la que se observa en la condición de línea de base y ante estímulos de risa menos contagiosaimplicó el análisis de las dos comparaciones contenidas en la hipótesis de forma independiente.

En este caso, dado que no todas las variables relacionadas con la comparación de intervalos $\mathrm{R}-\mathrm{R}$ entre línea de base y exposición a estímulos de risa más contagiosa tienen distribución normal (Kolmogorov-Smirnov: $p<.05$ ), la significación de las comparaciones de interés se estableció con la prueba no paramétrica de rangos de Wilcoxon (véase Tabla 3).

Como se puede observar, los valores de la Tabla 3 muestran que no existen diferencias significativas en el promedio de intervalos $\mathrm{R}-\mathrm{R}$ entre la condición de línea de base y durante la exposición a las risas femenina y masculina más contagiosas. Así, se puede afirmar que los datos reportados no apoyan este componente de la tercera hipótesis (H3) de esta investigación.

Adicional a esto, la comparación de intervalos R-R entre las condiciones de exposición a risas más y menos contagiosas se basó en los datos sobre las risas más contagiosas que se señalan en la Tabla 3 y en los siguientes datos sobre las risas menos contagiosas: risa femenina menos

Tabla 3.

Prueba de rangos de Wilcoxon de promedio de duración de intervalos $R-R$ de los participantes en línea de base y frente a los estímulos de risa femenina y masculina más contagiosa

\begin{tabular}{|c|c|c|c|c|c|}
\hline Condición experimental ${ }^{a}$ & $\begin{array}{c}\text { Promedio de duración } \\
\text { intervalos }{ }^{\mathrm{b}} \mathrm{R}-\mathrm{R}\end{array}$ & $\begin{array}{l}\text { Intervalos de con- } \\
\text { fianza } \\
\end{array}$ & $D T$ & Valor de Z & Significación \\
\hline Línea de base & 0.7387 & $0.7068-0.7707$ & 0.1237 & \multirow[b]{2}{*}{-1.119} & \multirow[b]{2}{*}{$p<0.05$} \\
\hline $\begin{array}{l}\text { Estímulo de risa femenina } \\
\text { más contagiosa }\end{array}$ & 0.7874 & $0.7271-0.8477$ & 0.2333 & & \\
\hline Línea de base & 0.7387 & $0.7068-0.7707$ & 0.1237 & \multirow[b]{2}{*}{-7.21} & \multirow[b]{2}{*}{$p<0.05$} \\
\hline $\begin{array}{l}\text { Estímulo de risa masculina } \\
\text { más contagiosa }\end{array}$ & 0.7293 & $0.6828-0.7758$ & 0.1800 & & \\
\hline
\end{tabular}

Nota. ${ }^{\mathrm{a}} n=60 .{ }^{\mathrm{b}}$ Fracciones de segundo. DT: desviación típica. 
contagiosa $(M E=0.7473, D T=12.11, I C: 0.71-0.77)$ y risa masculina menos contagiosa $(M E=0.7579, D T=0.1986$, $I C: 0.70-0.80$ ). Para establecer la significación de la diferencia observada entre las medias de los diferentes tipos de risa se utilizó un ANOVA de medidas repetidas — donde el factor intrasujeto fue el tipo de risa, y la variable de medida fue la duración promedio de intervalos R-R expresados en fracciones de segundo-. Dado que el valor de Mauchly para establecer esfericidad no fue significativo $(p<.05)$, se tomó la corrección de Huynh-Feldt, que mostró un valor no significativo de efectos intrasujetos $(F(2.42142 .80)=1.88$, $p>.05 n^{2}=0.031$ ).

Con base en los análisis estadísticos expuestos en el párrafo anterior, se puede afirmar que los datos de esta investigación no mostraron apoyo empírico para la tercera hipótesis (H3) de este estudio, por lo cual se puede afirmar que no hubo incrementos en la frecuencia cardíaca en exposiciones a estímulos de risa más contagiosos en comparación con la línea de base, ni mayores niveles de esta variable ante risas más contagiosas con respecto a las menos contagiosas.
Diferencias de sexo en los resultados relacionados con las hipótesis

Por último, para determinar las diferencias de sexo relacionadas con las hipótesis verificadas, y ante la imposibilidad de utilizar pruebas paramétricas — como un análisis factorial- por violación de varios supuestos, se utilizó la prueba no paramétrica de Wilcoxon. Así, se partió de la idea de que la exposición a las condiciones de risas femenina y masculina más contagiosas debían producir mayores niveles de expresiones emocionales en comparación con los valores observados en la línea base y ante las condiciones de risa menos contagiosa, lo cual implica observar diferencias al comparar las diversas condiciones. Teniendo esto en cuenta, se compararon los datos de hombres y mujeres sobre la diferencia de los niveles de expresión facial, EMG e intervalos R-R entre las condiciones de risas más contagiosas y línea de base y entre estas y las condiciones de risas menos contagiosas.

Como se puede apreciar en la Tabla 4, a excepción de un valor significativo, los participantes hombres y mujeres no difieren en los promedios de las diferencias entre las diversas expresiones emocionales comparadas.

Tabla 4.

Comparación por sexos de la diferencia en las expresiones emocionales provocadas entre las risas más contagiosas y la línea de base/risas menos contagiosas mediante la prueba Wilcoxon

\begin{tabular}{|c|c|c|c|c|}
\hline $\begin{array}{l}\text { Expresiones } \\
\text { emocionales }\end{array}$ & Comparaciones & Mujeres & Hombres & $\begin{array}{l}\text { Valor z y } \\
\text { significación }\end{array}$ \\
\hline \multirow{4}{*}{$\begin{array}{l}\text { Expresiones faciales } \\
\text { de alegría }\end{array}$} & $\begin{array}{l}\text { Diferencia promedio de porcentajes de alegría entre } \\
\text { exposición a risa femenina más contagiosa y línea } \\
\text { de base. }\end{array}$ & $\begin{aligned} N & =35 \\
M E & =0.33 \\
D T & =0.24\end{aligned}$ & $\begin{aligned} N & =18 \\
M E & =0.36 \\
D T & =0.21\end{aligned}$ & $\mathrm{z}=-5.44(p>0.05)$ \\
\hline & $\begin{array}{l}\text { Diferencia promedio de porcentajes de alegría entre } \\
\text { exposición a risa masculina más contagiosa y línea } \\
\text { de base. }\end{array}$ & $\begin{aligned} N & =33 \\
M E & =0.31 \\
D T & =0.22\end{aligned}$ & $\begin{aligned} N & =16 \\
M E & =0.25 \\
D T & =0.23\end{aligned}$ & $\mathrm{z}=-0.82(p>0.05)$ \\
\hline & $\begin{array}{l}\text { Diferencia promedio de porcentajes de alegría entre } \\
\text { exposición a risa femenina más y menos contagiosa. }\end{array}$ & $\begin{aligned} N & =34 \\
M E & =0.19 \\
D T & =0.18\end{aligned}$ & $\begin{aligned} N & =17 \\
M E & =0.23 \\
D T & =0.22\end{aligned}$ & $\mathrm{z}=-0.45(p>0.05)$ \\
\hline & $\begin{array}{l}\text { Diferencia promedio de porcentajes de alegría } \\
\text { entre exposición a risa masculina más y menos } \\
\text { contagiosa. }\end{array}$ & $\begin{aligned} N & =32 \\
M E & =0.08 \\
D T & =0.17\end{aligned}$ & $\begin{aligned} N & =15 \\
M E & =0.15 \\
D T & =0.18\end{aligned}$ & $z=-0.90(p>0.05)$ \\
\hline \multirow{4}{*}{ Amplitud EMG } & $\begin{array}{l}\text { Diferencia promedio de amplitud EMG entre exposicio- } \\
\text { nes a risa femenina más contagiosa y línea de base. }\end{array}$ & $\begin{aligned} N & =39 \\
M E & =0.03 \\
D T & =0.03\end{aligned}$ & $\begin{aligned} N & =21 \\
M E & =0.04 \\
D T & =0.05\end{aligned}$ & $\mathrm{z}=-1.40(p>0.05)$ \\
\hline & $\begin{array}{l}\text { Diferencia promedio de amplitud EMG entre exposicio- } \\
\text { nes a risa masculina más contagiosa y línea de base. }\end{array}$ & $\begin{aligned} N & =39 \\
M E & =0.02 \\
D T & =0.03\end{aligned}$ & $\begin{aligned} N & =21 \\
M E & =0.02 \\
D T & =0.02\end{aligned}$ & $\mathrm{z}=-0.36(p>0.05)$ \\
\hline & $\begin{array}{l}\text { Diferencia promedio de amplitud EMG entre exposicio- } \\
\text { nes a risa femenina más y menos contagiosa. }\end{array}$ & $\begin{aligned} N & =39 \\
M E & =0.01 \\
D T & =0.02\end{aligned}$ & $\begin{aligned} N & =21 \\
M E & =0.02 \\
D T & =0.04\end{aligned}$ & $\mathrm{z}=-0.29(p>0.05)$ \\
\hline & $\begin{array}{l}\text { Diferencia promedio de amplitud EMG entre exposicio- } \\
\text { nes a risa masculina más y menos contagiosa. }\end{array}$ & $\begin{array}{c}N=39 \\
M E=0.008 \\
D T=0.02\end{array}$ & $\begin{aligned} N & =21 \\
M E & =0.01 \\
D T & =0.02\end{aligned}$ & $\mathrm{z}=-1.7(p>0.05)$ \\
\hline
\end{tabular}




\begin{tabular}{|c|c|c|c|c|}
\hline $\begin{array}{l}\text { Expresiones } \\
\text { emocionales }\end{array}$ & Comparaciones & Mujeres & Hombres & $\begin{array}{l}\text { Valor z y } \\
\text { significación }\end{array}$ \\
\hline \multirow{4}{*}{ Intervalos R-R } & $\begin{array}{l}\text { Diferencia promedio de intervalos } \mathrm{R}-\mathrm{R} \text { entre exposicio- } \\
\text { nes a risa femenina más contagiosa y línea de base. }\end{array}$ & $\begin{aligned} N & =39 \\
M E & =0.08 \\
D T & =0.24\end{aligned}$ & $\begin{array}{c}N=21 \\
M E=-0.01 \\
D T=0.08\end{array}$ & $\mathrm{z}=-1.82(p>0.05)$ \\
\hline & $\begin{array}{l}\text { Diferencia promedio de intervalos R-R entre exposicio- } \\
\text { nes a risa masculina más contagiosa y línea de base. }\end{array}$ & $\begin{array}{c}N=39 \\
M E=-0.03 \\
D T=0.18\end{array}$ & $\begin{array}{c}N=21 \\
M E=-0.08 \\
D T=0.23\end{array}$ & $\mathrm{z}=-0.78(p>0.05)$ \\
\hline & $\begin{array}{l}\text { Diferencia promedio de intervalos } \mathrm{R}-\mathrm{R} \text { entre exposicio- } \\
\text { nes a risa femenina más y menos contagiosa. }\end{array}$ & $\begin{array}{c}N=39 \\
M E=-0.07 \\
D T=0.19\end{array}$ & $\begin{array}{c}N=21 \\
M E=-0.01 \\
D T=0.04\end{array}$ & $\mathrm{z}=-2.55(p>0.05)$ \\
\hline & $\begin{array}{l}\text { Diferencia promedio de intervalos R-R entre exposicio- } \\
\text { nes a risa masculina más y menos contagiosa. }\end{array}$ & $\begin{array}{c}N=39 \\
M E=-0.01 \\
D T=0.23\end{array}$ & $\begin{array}{c}N=21 \\
M E=-0.05 \\
D T=0.21\end{array}$ & $\mathrm{z}=-0.92(p>0.05)$ \\
\hline
\end{tabular}

\section{Discusión}

El propósito de esta investigación fue determinar si los estímulos acústicos de risa contagiosa, además de generar conductas de risa o sonrisa (Provine, 1992), provocan en los oyentes las expresiones faciales, electromiográficas y cardíacas de una emoción positiva. La idea era aportar elementos para la comprensión de la naturaleza de esta risa $\mathrm{y}$ de las respuestas que provoca, y los hallazgos reportados en la sección anterior responden a este objetivo, incluso desde diferentes modalidades de respuesta emocional, lo cual permite establecer la convergencia de los resultados y la posibilidad de fortalecer la validez de las implicaciones formuladas.

En particular, respecto a la primera hipótesis $(\mathrm{H} 1)$ de este estudio — en la que se estableció que la exposición de los participantes del estudio a estímulos acústicos de risa más contagiosa provoca mayores expresiones faciales de alegría en comparación con las observadas en línea de base y ante estímulos de risa menos contagiosa-, los datos apoyaron esta suposición tanto en el caso de risa femenina como masculina, pues no solo se comprobó que la exposición a un estímulo de risa contagiosa produce una emoción positiva que no era evidente o aparecía con menor intensidad en la condición de línea de base, sino que fue claro que los estímulos de risa más contagiosa generan mayor porcentaje de microexpresiones de alegría que los menos contagiosos.

Relacionado con esto último, el software FaceReader registra variaciones de intensidad en las microexpresiones emocionales provocadas por diferentes estímulos (Terzis et al., 2011), de forma que la intensidad de aquellas se relacionaría con la capacidad de estos para provocar cambios en la expresión facial emocional. Con base en este razonamiento, se puede afirmar que los resultados respecto a la primera hipótesis de este estudio también indicarían que las variaciones en la intensidad de las expresiones de alegría serían proporcionales al grado de contagio percibido en las risas escuchadas.

Ahora bien, el resultado de esta hipótesis es especialmente relevante para el objetivo de esta investigación, pues la expresión facial se considera el medio más confiable para identificar las emociones que se expresan de forma automática (Maison \& Pawlowska, 2017). Así, con base en este hallazgo, hay razones para considerar que las respuestas provocadas por risas contagiosas tendrían una naturaleza emocional positiva. Incluso, este hallazgo resulta interesante debido a que, aunque la investigación de las últimas décadas ha profundizado en las diferentes formas de expresión emocional de la risa espontánea (p. ej., Gervais \& Wilson, 2005; Szameitat et al., 2009b), a excepción de algunos estudios que han profundizado en determinados correlatos emocionales de las diversas risas (p. ej., Szameitat et al., 2009a; Szameitat et al., 2009b; Szameitat et al., 2010; Wood et al., 2017), la investigación es escasa sobre la naturaleza y expresión emocional de estas.

En cuanto a la risa contagiosa, el estudio de Provine (1992) señaló varias respuestas psicofisiológicas que presentaron los participantes expuestos a estímulos de esta modalidad de risa, que el autor no relacionó con emociones. Por el contrario, la presente investigación ofrece evidencia de respuestas faciales de valencia emocional positiva provocadas por esta vocalización, las cuales mantendrían interrelación con otros canales de respuesta.

La probable naturaleza emocional positiva de las expresiones de sonrisa/risa provocadas por la risa más contagiosa basada en la comprobación de esta hipótesis tiene varias implicaciones para la teoría de contagio emocional (Hatfield et al., 2014):

a. Algunos autores (p. ej., Weber \& Quiring, 2019) han formulado como hipótesis que la risa contagiosa 
puede considerarse como un caso de contagio emocional primitivo (Hatfield et al., 2014); sin embargo, hasta la fecha no se contaba con datos empíricos que respaldaran esta suposición. Los hallazgos de este estudio ofrecen una base empírica para afirmar que este tipo de risa no solo tiene la capacidad de provocar respuestas de contagio vocales o motoras, sino también emocionales. Obviamente, se pueden esperar diferencias individuales en la capacidad de los receptores de esta risa para experimentar contagio emocional (Niedenthal \& Ric, 2017).

b. Teniendo en cuenta que el contagio emocional implica que la emoción que experimenta el emisor es la misma que expresa el receptor (Hess et al., 2014), se puede formular la hipótesis de que la alegría que experimentan los receptores de risa contagiosa es la misma emoción que transmiten los emisores de esta vocalización. Esta situación explica la convergencia y acoplamiento emocional y conductal que produciría esta risa entre el emisor y el receptor de la misma (Gervais \& Wilson, 2005; Scott et al., 2014), la cual favorecería la cooperación, cohesión y consecución de metas comunes (Owren \& Bachorowsky, 2001).

c. Según la teoría de contagio emocional (Hatfield et al., 2014), este proceso se iniciaría con la imitación motora de la expresión emocional del emisor; sin embargo, en esta investigación los participantes no tenían una expresión facial para imitar, pues solo fueron expuestos a audios de risa. Teniendo esto en cuenta, los resultados de este estudio confirman la posibilidad de contagio emocional provocado exclusivamente por vocalizaciones, lo que generaría cambios concomitantes en la expresión facial del receptor congruentes con la emoción contagiada.

Por otra parte, los resultados relacionados con la segunda hipótesis (H2) de este estudio — respecto a que la exposición de los participantes a estímulos de risa más contagiosa provoca mayor actividad EMG del músculo facial cigomático en comparación con la que se observa en la condición de línea de base y ante estímulos de risa menos contagiosa - apoyaron dicha suposición tanto para risas femeninas como masculinas; resultado que implica que las risas más contagiosas serían estímulos competentes para provocar contracciones y actividad eléctrica registrable en el cigomático mayor, y que la actividad EMG de este músculo variaría según el grado de contagio percibido de los estímulos de risa contagiosa.

Los resultados relacionados con esta hipótesis también son relevantes para el objetivo general de esta investigación, dada la alta sensibilidad de la señal EMG y su capacidad para determinar no solo la valencia afectiva, sino también la intensidad de de la respuesta emocional (Hess, 2009). De hecho, aunque se ha comprobado que la actividad EMG facial no discrimina con exactitud la emoción positiva que experimenta un individuo (Boxtel, 2010), en varias investigaciones se ha relacionado la mayor contracción y actividad eléctrica del músculo cigomático con afectos y emociones positivas (p. ej., Hess, 2009). De esta forma, los resultados relacionados con la segunda hipótesis de este estudio confirmarían la naturaleza emocional positiva de las reacciones emocionales que presentaron los participantes expuestos a estímulos de risa contagiosa.

Adicional a esto, con base en los resultados relacionados con esta hipótesis, es posible corroborar que las risas más contagiosas provocan respuestas emocionales positivas y de mayor intensidad que las menos contagiosas. La literatura sobre el tema (p. ej., Gervais \& Wilson, 2005) señala que las risas acompañadas por expresiones emocionales más intensas tenderían a ser percibidas como espontáneas y generadoras de nuevos contagios, lo cual explicaría las cadenas de contagio de risa que se observan en la vida cotidiana cuando una persona que presenta risa provocada contagia a los demás miembros de un grupo.

Por otra parte, la tercera hipótesis (H3) de esta investigación afirmaba que la exposición de los participantes a estímulos de risa más contagiosa provoca mayor frecuencia cardíaca - menor duración de los intervalos R-R - que la que se observa en la condición de línea de base y ante estímulos de risa menos contagiosa, pero los resultados relacionados con esta hipótesis no apoyaron esta suposición en ninguna de las condiciones evaluadas. Este hallazgo implica que las risas más contagiosas no provocarían cambios en la frecuencia cardíaca concomitantes con las demás respuestas de expresión emocional provocadas, y que bajo las condiciones en las que aplicó este experimento y se obtuvieron los datos de la variable dependiente, los intervalos R-R no permitieron detectar diferentes respuestas cardíacas ante estímulos de risa con diverso grado de contagio percibido.

Los resultados relacionados con esta hipótesis implican tres posibles interpretaciones: (a) que la exposición a estímulos de risa más contagiosa, tal como se realizó en este experimento, no afectaría la frecuencia cardíaca de forma significativa; (b) que el indicador de frecuencia cardíaca utilizado en este estudio (intervalos R-R) no sería sensible a las variaciones emocionales provocadas por los estímulos utilizados; y (c) que, en contraste con lo esperado en este estudio, como respuesta a los estímulos de risa más contagiosa predominaron las sonrisas sobre las risas, y la literatura señala una menor frecuencia cardíaca en la 
emisión de las primeras con respecto a las segundas (p. ej., Domínguez, 2013).

Adicional a esto, los resultados relacionados con esta hipótesis se puedan atribuir a la dificultad que tendrían los investigadores para identificar la activación simpática específica elicitada por emociones positivas como la alegría (Herring et al., 2011). Al contrario de lo que sucede con las emociones negativas, las positivas presentan algunas expresiones fisiológicas menos intensas y salientes que no siempre son detectadas por los instrumentos de medición psicofisiológica (Domínguez, 2013).

Finalmente, respecto a las diferencias entre sexos, en términos generales los resultados mostraron concordancia en los valores promedio de las expresiones emocionales provocadas por las risas más contagiosas femeninas o masculinas en los participantes de ambos sexos. Este hallazgo, además de ratificar los resultados del primer estudio de Arévalo (2020), quien no encontró diferencias de sexo en la apreciación de la risa contagiosa, sugiere que tanto hombres como mujeres estarían preparados biológicamente para responder de manera análoga ante este tipo de vocalización. Así, desde un punto de vista evolucionista, podría pensarse que esta risa de origen filogenéticamente antiguo (Provine, 1992) tiene la capacidad adaptativa de lograr sintonía y convergencia social independientemente del sexo de quien la emita o la escuche.

Ahora bien, integrando los resultados observados sobre las tres hipótesis, se puede afirmar que dada la alta confiabilidad que se le atribuye a la expresión facial y a la EMG facial en el reconocimiento de emociones (Hess, 2009; Maison \& Pawlowska, 2017) y a la convergencia de los resultados observados en las dos primeras hipótesis de esta investigación, es posible señalar la alta probabilidad de que la risa contagiosa provoque emociones positivas en los oyentes, aunque esta conclusión se formula con cautela por los resultados desconfirmatorios relacionados con la actividad cardíaca.

Asimismo, los hallazgos que confirman la naturaleza emocional positiva de las respuestas provocadas de risa o sonrisa permiten suponer que la exposición a estímulos de risa contagiosa activarían el sistema endógeno opiode de los receptores, lo cual se relaciona con emociones positivas e importantes para la vinculación y la comunicación prosocial en primates humanos y no humanos (Nummenmaa \& Tuominen, 2018). Así, la suspuesta activación de este sistema por parte de estímulos de risa contagiosa fomentaría las conductas y efectos sociales atribuidos a este tipo de risa (Gervais \& Wilson, 2005).
Por último, a pesar de los aportes de este estudio, es necesario reconocer algunas limitaciones y debilidades que enfrentó la presente investigación:

a. Dada la escasez de estudios sobre el tema, esta investigación no tuvo suficientes antecedentes teóricos y empíricos que facilitaran su diseño e implementación. Teniendo esto en cuenta, el presente estudio constituye un abordaje exploratorio sobre esta modalidad de risa.

b. En este estudio no se registraron señales electrofisiológicas adicionales que hubieran podido aportar información confirmatoria sobre la naturaleza emocional de las respuestas provocadas por los audios de risa contagiosa. En este orden de ideas, hubiera sido deseable recolectar datos sobre la frecuencia respiratoria y la respuesta electrodérmica de los participantes expuestos a diferentes estímulos de esta vocalización.

c. Este estudio no registró la actividad EMG del músculo orbicular del párpado, que hubiera podido confirmar o desconfirmar la naturaleza emocional positiva de las respuestas faciales provocadas (Korb et al., 2014).

d. A pesar de que este estudio se aplicó en un laboratorio relativamente aislado, las mediciones EMG y EMG son susceptibles a interferencias por ruidos o eventos externos (p. ej., Huang et al., 2005; Orini et al., 2011) o a ser afectadas por procesos no emocionales o no relacionados con la risa.

e. El presente estudio no solicitó a los participantes reportes subjetivos de las emociones experimentadas frente a los diversos estímulos de risa, los cuales se hubieran podido contrastar con la información objetiva recolectada.

f. Por ser este un estudio de laboratorio que utilizó una muestra por conveniencia, los resultados de esta investigación pueden tener alta validez interna, pero baja validez externa.

Para estudios futuros y aplicaciones prácticas sobre el tema de esta investigación, se formulan las siguientes recomendaciones:

a. Dado que la risa y las emociones positivas se han relacionado con salud, satisfacción, bienestar, mejor capacidad de afrontamiento, creatividad, etc. (p. ej., Mora \& Quintana, 2010; Tugade et al., 2004), y que a la fecha no se conocen los beneficios específicos asociados a la risa contagiosa, se sugiere investigar respecto a la capacidad de este tipo de risa para provocar emociones positivas en los oyentes, así 
como la posibilidad de utilizarla en el campo de la salud preventiva o curativa.

b. Dado que en la presente investigación predominaron las respuestas provocadas de sonrisa sobre las de risa, lo cual no permitió contar con una muestra representativa de audios de esta vocalización, se sugiere aplicar estudios que caractericen acústicamente las risas provocadas por risas contagiosas, con el fin de determinar su naturaleza emocional vocal.

c. Como otras posibles aplicaciones prácticas de los hallazgos de esta investigación, se sugiere, por una parte, que la risa contagiosa y su capacidad para provocar emociones podría ser útil en comunicaciones persuasivas en las que se pueden asociar mensajes emocionalmente neutros para una audiencia con estímulos acústicos de esta vocalización, con el fin de lograr modificación actitudinal y emocional hacia el contenido de los mismos; $y$, por otra, que el conocimiento sobre la risa contagiosa y sus efectos emocionales puede ayudar a mejorar la selección y efectividad de las risas grabadas que se utilizan en los programas humorísticos de televisión o radio para aumentar la percepción de comicidad de los contenidos presentados.

En conclusión, este estudio aporta los siguientes elementos para la comprensión de las respuestas de risa/sonrisa elicitadas por la risa contagiosa: (a) las respuestas provocadas por este tipo de vocalización pueden ser consideradas como expresiones emocionales de valencia positiva; (b) se observa proporcionalidad entre el grado de contagio percibido de los estímulos de risa y la intensidad de las respuestas emocionales provocadas; (c) el efecto de contagio emocional de la risa contagiosa se produce independientemente de la familiaridad o historia de interacción o reforzamiento entre el emisor y el receptor de esta vocalización; y (d) los resultados de este estudio apoyarían la teoría de que la función principal de la risa es provocar emociones y afectos positivos en los receptores (Owren \& Bachoroswski, 2003).

\section{Declaración de ausencia de conflicto de intereses}

Los autores del presente artículo declaran no tener conflicto de interés alguno para la presentación y publicación del mismo.

\section{Referencias}

Arévalo, G. (2020). Parámetros acústicos de la risa contagiosa como estímulo incondicionado en condicionamiento de actitudes hacia la marca (Tesis doctoral no publicada). Programa de Psicología, Universidad de los Andes, Bogotá, Colombia.
Blanca, M. J., Alarcón, R., Arnau, J., Bono, R., \& Bendayan, R. (2017). Non-normal data: Is ANOVA still a valid option? Psicothema, 29(4), 552-557. https://doi.org/10.7334/ psicothema2016.383

Biopac Systems, Inc. (2016). Tutorial básico. Manual Revision 4 (01.07.2016). https://www.biopac.com/wp-content/uploads /bsl-4-tutorial-es.pdf

Boxtel, A. (2010). Facial EMG as a tool for inferring affective states (conference paper). En A. J., Spink, F., Grieco, O. E., Krips, L. W. S., Loijens, L. P. J. J. Noldus, \& P. H., Zimmerman (Eds.), Proceedings of measuring behavior (pp. 104-108). Eindhoven, The Netherlands, 17-24 August. Wageningen: Noldus Information Technology. https://www. measuringbehavior.org/files/ProceedingsPDF(website)/ Boxtel_Symposium6.4.pdf

Buchowski, M. S., Majchrzak, K. M., Blomquist, K., Chen, K. Y., Byrne, D. W., \& Bachorowski, J-A. (2007). Energy expenditure of genuine laughter. International Journal of Obesity, 31, 131-137. https://doi.org/10.1038/sj.ijo.0803353

Cosentino, S., Sessa, S., \& Takanishi, A. (2016). Quantitative laughter detection, measurement, and classification-Acritical survey. IEEE Reviews in Biomedical Engineering, 9, 1-15. https://ieeexplore.ieee.org/stamp/stamp.jsp?tp=\&arnum ber $=7403873$

Domínguez, F. J. (2013). La alegría, la tristeza y la ira. En E. G. Fernández-Abascal, B. García, M. P. Jiménez, M. D. Martín \& F. J. Domínguez (Eds.), Psicología de la emoción (pp. 267-238). Editorial Universitaria Ramón Areces.

Gervais, M., \& Wilson, D. S. (2005). The evolution and functions of laughter and humor: a syntethic approach. The Quarterly Review of Biology, 80(4), 395-430. https://doi. org/10.1086/498281

Hatfield, E., Bensman L., Thornton P. D., \& Rapson R. L. (2014). New perspectives on emotional contagion: A review of classic and recent research on facial mimicry and contagion. Interpersona: An International Journal of Personal Relationships, 8(2), 159-179. https://pdfs.semanticscho lar.org/74d9/c58c2f238b66a8f842801e14aa3d3406c250. pdf?_ga=2.189937067.189994778.1618796988-5145659 6.1557093542

Herring, D. R., Burleson, M., Roberts, N., \& Devine, M. J. (2011). Coherent with laughter: Subjective experience, behavior, and physiological responses during amusement and joy. International Journal of Psychophysiology, 79(2), 211218. https://doi.org/10.1016/j.ijpsycho.2010.10.007

Hess, U. (2009). Facial EMG. En E. Harmon-Jones \& J. S. Beer (Eds.), Methods in social science (pp. 70-148). The Guilford Press. https://books.google.com.co/books?hl=es\&lr=\&id $=\mathrm{P} 004 \mathrm{DqMBmrYC} \& \mathrm{oi}=\mathrm{fnd} \& \mathrm{pg}=\mathrm{PA} 70 \& \mathrm{dq}=\mathrm{Hess},+\mathrm{U}$. $+(2009) .+$ Facial + EMG + pdf\&ots $=$ Sa3ZsEllqj\&sig $=$ GNVK2 rmNM-4MNuCzeHcNi0sWySk\#v=onepage\&q\&f=false

Hess, U., Houde, S., \& Fischer, A. (2014). Do we mimic what we see or what we know? En C. von Scheve, \& M. Salmela 
(Eds.), Collective emotions (pp. 94-107). Oxford University Press.

Hietanen, J. K., Surakka, V., \& Linnankoski, I. (1998). Facial electromyographic responses to vocal affect expressions. Psychophysiology, 35(5), 530-536. https://doi.org/10.1017/ S0048577298970445

Huang, C-N., Cheng, C-H., \& Chung, H-Y. (2005). The review of applications and measurements in facial electromyography. Journal of Medical and Biological Engineering, 25(1), 15-20. https://citeseerx.ist.psu.edu/viewdoc/download?doi $=10.1 .1 .731 .5672 \& \mathrm{rep}=\mathrm{rep} 1 \&$ type $=$ pdf

Korb, S., With, S., Niedenthal, P., Kaiser, S., \& Grandjean, D. (2014). The perception and mimicry of facial movements predict judgments of smile authenticity. PLOS ONE, 9(6), e99194. https://doi.org/10.1371/journal.pone.0099194

Kreibig, S. D. (2010). Autonomic nervous system activity in emotion: A review. Biological Psychology, 84(3), 394-421. https://doi.org/10.1016/j.biopsycho.2010.03.010

Maison, D., \& Pawlowska, B. (2017). Using the Facereader method to detect emotional reaction to controversial advertising referring to sexuality and homosexuality. En K. Nermend, \& M. Latuszyn'ska (Eds.), Neuroeconomic and behavioral aspects of decision making (pp. 309-327). Springer Proceedings in Business and Economics. https:// link.springer.com/chapter/10.1007/978-3-319-62938-4_20

Mathôt, S., Schreij, D., \& Theeuwes, J. (2012). OpenSesame: An open-source, graphical experiment builder for the social sciences. Behavior Research Methods, 44(2), 314-324. https://link.springer.com/content/pdf/10.3758/s13428-011 -0168-7.pdf

Matsumoto, D. (1992). American-japanesse cultural differences in the recognition of universal facial expressions. Journal of Cross-cultural Psychology, 23(1), 72-84. http://matsumoto group.com/content/1992\%20American\%20Japanese $\% 20$ Cultural\%20Differences.pdf

Matsumoto, D., Keltner, D., Shiota, M. N., O'Sullivan, M., \& Frank, M. (2008). Facial expressions of emotion. En M. Lewis, J. M. Haviland-Jones \& L. F. Barret (Eds.), Handbook of emotions ( $3{ }^{a}$ ed., pp. 211234). Guilfod Press. https://www.researchgate.net/ publication/284387911_Facial_expressions_of_emotion

Mauss, I. B., Levenson, R. W., McCarter, L., Wilhelm, F. H., \& Gross, J. J. (2005). The tie that binds? Coherence among emotion experience, behavior, and physiology. Emotion, 5(2), 175-190. https://doi.org/10.1037/1528-3542.5.2.175

Mora, R., \& Quintana, I. (2010). Laughter and positive therapies: Modern approach and practical use in medicine. Revista de Psiquiatría y Salud Mental, 3(1), 27-34. https:// www.elsevier.es/en-revista-revista-psiquiatria-salud-men tal-486-pdf-S2173505010700066

Neves, L., Cordeiro, C., Scott, S., Castro, S. L., \& Lima, C. (2018). High emotional contagion and empathy are associated with enhanced detection of emotional authenticity in laughter.
Quarterly Journal of Experimental Psychology, 71(11), 2355-2363. https://doi.org/10.1177/1747021817741800

Niedenthal, P. M., \& Ric, F. (2017). Psycholgy of emotion: interpersonal, experential and cognitive approaches (2. ${ }^{\mathrm{a}} \mathrm{ed}$.). Routedge.

Nummenmaa, L., \& Tuominen, L. (2018). Opioid system and human emotions. British Journal of Pharmacology, 175(14), 2737-2749. https://doi.org/10.1111/bph.13812

Orini, M., Peláez-Coca, M. D., Bailón, R., \& Gil, E. (2011). Estimation of spontaneous respiratory rate from photoplethysmography by cross time-frequency analysis. Computing in Cardiology, 38, 661-664. http://cinc.mit.edu/ archives/2011/pdf/0661.pdf

Owren, M. J., \& Bachorowski, J. A. (2001). The evolution of emotional expression: A "selfish-gene" account of smiling and laughter in early hominids and humans. En T. J. Mayne \& G. A. Bonanno (Eds.), Emotion: Current issues and future directions (pp. 152-191). The Guilford Press. https:// www.academia.edu/523109/The_evolution_of_emotio nal_expression_A_selfish_gene_account_of_smiling_and laughter_in_early_hominids_and_humans?auto=download

Owren, M. J., \& Bachorowski, J. A. (2003). Reconsidering the evolution of nonlinguistic communication: The case of laughter. Journal of Nonverbal Behavior, 27, 183-200.

Owren, M. J., Philipp, M. C., Vanman, E., Trivedi, N., Schulman, A., \& Bachorowski, J. A. (2013). Understanding spontaneous human laughter: The role of voicing in inducing positive emotion. En E. Altenmüller, S. Schmidt, \& E. Zimmermann (Eds.), Evolution of emotional communication: From sounds in nonhuman mammals to speech and music in man (pp. 176-190). Oxford University Press. https:// doi.org/10.1093/acprof:oso/9780199583560.003.0011

Provine, R. R. (1992). Contagious laughter: laughter is a sufficient stimulus for laughs and smiles. Bulletin of Psychonomic Society, 30(1), 1-4. https://link.springer.com/ content/pdf/10.3758/BF03330380.pdf

Provine, R. R. (2015). Laughter as a scientific problem: An adventure in sidewalk neuroscience. The Journal of Comparative Neurology, 524(8), 1532-1539. Wiley Online Library. https://doi.org/10.1002/cne.23845

Resolución 008430 de 1993. Por la cual se establecen las normas científicas, técnicas y administrativas para la investigación en salud. Bogotá. Ministerio de Salud. https://www. minsalud.gov.co/sites/rid/Lists/BibliotecaDigital/RIDE/ DE/DIJ/RESOLUCION-8430-DE-1993.PDF

Ruef-López, R., Navarro, J., Caetano, A., \& Silva, A. (2015). A Markov chain analysis of emotional exchange in voice-tovoice communication: Testing for the mimicry hypothesis of emotional contagion. Human Communication Research, 41(3), 412-434. https://doi.org/10.1111/hcre.12051

Scott, S. K., Lavan, N., Chen, S., \& McGettigan, C. (2014). The social life of laughter. Trends in cognitive sciences, 18(12), 618-620. https://doi.org/10.1016/j.tics.2014.09.002 
Shaughnessy, J. J., Zeichmeister, E. B., \& Zeichmeister, J. S. (2007). Métodos de investigación en Psicología. McGraw-Hill.

Szameitat, D. P., Alter, K., Szameitat, A. J., Darwin, C. J, Wildgruber, D., Dietrich, S., \& Sterr, A. (2009a). Differentiation of emotions in laughter at the behavioral level. Emotion, 9, 397-405. https://doiorg/10.1037/a0015692

Szameitat, D. P., Alter, K., Szameitat, A. J., Wildgruber, D., Sterr, A., \& Darwin, C. J. (2009b). Acoustic profiles of distinct emotional expressions in laughter. The Journal of the Acoustical Society of America, 126(1), 354-366. https://doi. org/10.1121/1.3139899

Szameitat, D. P., Kreifelts, B., Alter, K., Szameitat, A. J., Sterr, A., Grodd, W., \& Wildgruber, D. (2010). It is not always tickling: Distinct cerebral responses during perception of different laughter types. NeuroImage, 53, 1264-1271. https:// doi.org/10.1016/j.neuroimage.2010.06.028

Terzis, V., Moridis, C., \& Economides, A. (2011). Measuring instant emotions based on facial expressions during computer based assessment. Personal and Ubiquitous Computing, 17(1). http://195.251.210.217/conta/publica tions/PDF/2011-\%20PUC-\%20Measuring\%20instant $\% 20$ emotions $\% 20$ based $\% 20$ on $\% 20$ facial $\% 20$ expressions $\% 20$ during\%20computer-based\%20assessment.pdf
Tugade, M. M., Fredrickson, B. L., \& Barrett, L. F. (2004). Psychological resilience and positive emotional granularity: Examining the benefits of positive emotions on coping and health. Journal of Personality, 72(6), 1161-1190. http:// dx.doi.org/10.1111/j.1467-6494.2004.00294.x

Weber, M., \& Quiring, O. (2019). Is it really that funny? Laughter, emotional contagion, and heuristic processing during shared media use. Media Psychology, 22(2), 173-195. https://doi.org/10.1080/15213269.2017.1302342

Whalen, P. K. (2010). The emotional and physiological structure of laughter: A comparison of three kinds of laughs (Antiphonal, Duchenne, and Voiced) and individual differences in the use of laughter in middle-aged and older marriages (Doctoral dissertation of Philosophy in Psychology). University of California, Berkeley. https:// digitalassets.lib.berkeley.edu/etd/ucb/text/Whalen berkeley_0028E_10301.pdf

Wood, A., Martin, J., \& Niedenthal, P. (2017). Towards a social functional account of laughter: Acoustic features convey reward, affiliation, and dominance. PLoS ONE, 12(8), e0183811. https://doi.org/10.1371/journal.pone.0183811 